\title{
Experimental Analysis of Browser based Novel Anti-Phishing System Tool at Educational Level
}

\author{
Rajendra Gupta \\ BSSS Autonomous College, Barkatullah University, Bhopal - 462024, India \\ E-mail: rajendragupta1@yahoo.com \\ Piyush Kumar Shukla \\ University Institute of Technology, Rajiv Gandhi Technical University, Bhopal - 462026, India \\ E-mail: pphdwss@gmail.com
}

\begin{abstract}
In the phishing attack, the user sends their confidential information on mimic websites and face the financial problem, so the user should be informed immediately about the visiting website. According to the Third Quarter Phishing Activity Trends Report, there are 55,282 new phishing websites have been detected in the month of July 2014. To solve the phishing problem, a browser based add-on system may be one of the best solution to aware the user about the website type. In this paper, a novel browser based add-on system is proposed and compared its performance with the existing antiphishing tools. The proposed anti-phishing tool 'ePhish' is compared with the existing browser based antiphishing toolbars. All the anti-phishing tools have been installed in computer systems at an autonomous college to check their performance. The obtained result shows that if the task is divided into a group of systems, it can give better results. For different phishing features, the add-on system tool show around 97 percentage successful results at different case conditions. The current study would be very helpful to countermeasure the phishing attach and the proposed system is able to protect the user by phishing attacks. Since the system tool is capable of handling and managing the phishing website details, so it would be helpful to identify the category of the websites.
\end{abstract}

Index Terms - Web browser, Add-on, Phishing, Antiphishing, Phishing Indicators.

\section{INTRODUCTION}

To reduce the phishing attack, it is necessary to make awareness among the web user about the type of websites and spread the message to the web user that how the phishing website steal the confidential information of the web user. The web browser is used to access the websites so the web browser based solution can be helpful to the web user to protect their confidential information from phishing attack. The web browser can directly warn the user about the type of website with the help of add-on which is an optional tool installed on it. This solution is more effective than other solutions for protection from phishing attack. In addition, the web browser market is mostly using three browsers i.e. Internet Explorer, Mozilla Firefox and Google Chrome which comprises around $90 \%$ of the total web browsers use [1]. So these web browsers taken for the testing and finding the result at education institute. The study of S. Egelman et.al [2] shows that when Firefox 2 web browser shows the phishing warnings on its display, none of the users entered sensitive information into the websites. The same study recommended that the result analysis of Internet Explorer's phishing warning. On the basis of research study, regular updations are going on with the web browser and they are giving effective results for the phishing countermeasures. It is necessary that the web browser should accurately identify the phishing web sites (low false positive result) so that the user can trust on the web browser's warning messages. Some web browsers are already providing the alert system for possible malicious attacks. If the website is not having HTTPs protocol and the user is feeding their credential information on it, the web browser should display the alert message to the user about the possible phishing attack. If the website is suspicious then the web browser checks the security certificate whether it is present in the website or not. After checking the security certificate, the web browser alerts the user about the type of website. To check the performance of anti-phishing tools, a research study has been done at an educational institute. The concept behind the designing of the Anti-phishing tool is that when internet user hit the URL, a dialog box appear on the screen that inform the user about the type of the website whether it is phishing or not. In the proposed add-on, the system is divided into five different assigned groups and the performance of the system tool is tested by data mining algorithms.

\section{RELATED WORK ON BROWSER BASED ANTI-PHISHING TOOL}

In the previous study, researchers has suggested and studied a number of anti-phishing system models to find the solution of phishing [3-9]. The earlier proposed models do not give more than 90 percentage successful result [10-14]. In some cases, the system tools are giving only 50-60 percentage successful result. Since the 
techniques and tools are upgrading day-by-day and changes are being happening in the website designing, so the web developer tries to utilize advanced techniques to make the phishing website. In this case, the existing tools are not finding accurate result. So it is noted that a system should be developed that can manage and support the advanced tools of web development so that the better result could be achieved. A. Martin et.al. [15] have worked on the 27 phishing criteria using the concept of Neural Network. The same criteria have been taken by other researchers to find the solution from phishing attack [16-20]. A survey on the anti-phishing techniques has been done which is helpful in this study [32].

\section{RESEARCH CRITERIA OF URL, CONTENT AND IMAGE MATCHING}

When web user wants to access the website, he first hit the web address on the URL or reached to the target webpage from any other website reference tags. In this case, first of all the URL and its contents should be checked then the contents and existing images should be checked [21]. To check the various indicators of the website, it takes several times to cross check the website information with the database information stored in the database of the Add-on. In the earlier study, browserbased client-side solutions have been proposed to mitigate the phishing attacks [22, 23]. Some techniques have also been developed which attempt to prevent phishing mails which are being delivered [24, 25]. So we should have a system that can fast and accurate check the fed information with the database information. To make the fast accessing system, I have defined the study points for the best possible solution. The studied criteria for the phishing have collected from the previous study [26, 27, 28]. Following are the study points of phishing criteria and the reason for taking these study points are discussed herewith.

\section{Number of dots '?' present in the URL}

When a website prepared, generally two '.' are used with the separation of www and the domain type. (e.g. www.mypage.com). If more number of '.' are using in the website, it means the attacker is trying to redirect the website to another webpage or trying to spoof the internet user. So if we found that the website is using more than 3 dots, the system can inform the user that 'It may be a risky site, don't feed any confidential information in it without confirmation'. If number of dot is more than 4, the system can declare the website is phishing. The example of phishing website is http://www.myhomepage.co.in//yahoo.co/php http://www.myhomepage.co.in/ login.php etc.

\section{Number of '@' present in the URL}

Some of the phishing attack uses '@' symbol to redirect the user to another website. Generally @ symbol is used in the FTP server to redirect the user. Since when user create his e-mail account, @ symbol is used. So the use of @ symbol in the URL is very good thinking of the attacker to spoof the web user. The attacker can create the website like http://www.myhomepage.co@yahoo.com?login.com. In this case user can think that he is directing from yahoo.com website.

\section{Number of '//' present in the URL}

When website prepares, it is uploaded with either http or https protocol. http protocol uses '//' symbol to redirect the webpage. So the phishing attack uses a number of '//' in the URL to spoof the web user. It is noticed that legitimate website do not uses more than two '//' symbol while redirecting the webpage. So if an attacker uses more than two '//' symbols, we can identify the whether the website is spoofing or not.

\section{Existence of IP (Internet Protocol) address in the URL}

In the functioning of any website, an IP address is provided to the domain of concerned URL. The sending and receiving of the data from the website functions with the use of this IP address. To spoof the user, generally attackers try to use IP address in URL instead of giving any alphabetic name. IPv4 addresses are separated in four different parts with the help of dot (.). For example http://www.84.214.244.122 instead of http://www.mywebpage.com

In such situation the internet user doesn't understand which website he is visiting.

\section{Port Number in the URL}

Some of the phishing URL try to redirect the web user to different port addresses. To do this attacker uses the target port number in its phishing URL address. For example the phishing website http://www.191.102.34.09:8087/http://myhomepage.co.in /index.htm trying to send the myhomepagewebsite contents to 8087 port of the server. Generally server has assigned 80 or 8080 port number. By tracing the port number from the URL address, we can find the website is trying to spoofing the internet user or not.

\section{The websites which are having HTTPs protocol}

It is noticed that phishing attack tries to make almost similar website to the legitimate website by ignoring the security. The phishing attack gives the attention for the changing of URL address, website contents, images etc. Since the security certificate is required to safe transaction over the web, the website holder takes the prior permission from the authority concern. When the authority gives the security permission to the website holder, the protocols converts with HTTPs. The website which uses HTTPs protocol can transfer the data securely. The phishing attack creates the spoofed URL address by ignoring the HTTPs. For example in place of https://www.google.com, the attacker can create the website http://www.gooogle.com

If attackers try to use fake security certificate in the website, web browser automatically detect the fake 
certificate and do not give the permission to the website to function.

\section{Number of Phishing Keywords present in the URL}

It is seen that some phishing attack uses phishing keywords in place of legitimate website contents by changing, replacing, shifting or deleting the characters from the website. For example in place of http://www.google.com, phishing attack can create the websitehttp://www.gooogle.com,http://www.goog1e.com, http://www.gugle.com etc. In this case suppose a user hit the wrong URL, he will send his confidential information to a spoofed website.

\section{Country Code present in the URL}

While checking the URL, country code with the help of WorldIP plug-in of Mozilla Firefox web browser, it is found that the URL web address doesn't match the exact country which is mentioned in the web URL. It is seen in the report of Advanced Phishing Working Group that some targeted countries country codes are used for web URL to lure the user. By cross-checking the country code and the IP address of the website, it can be determined that the user accessed website is legitimate or phishing.

\section{Title Tag}

Phishing websites generally do not emphasize on the title of the website. It is seen that sometimes in the phishing sites, the web address and the title tags remains different. For example the website http://www.derezo.com/kf06/ppl/paypal.html is a phishing website which uses the legitimate website title tag and tries to redirect the user to paypel website as target.

\section{Form Tags on the web page}

The Form tags are commonly used for the preparation of the website. It can be used for requesting user to feed the data into website. For example the form tag can be used for asking the information like login, password, credit card number etc. Mostly the phishing website developer uses the same form tags and fields to spoof the user. So by finding the number of Form tags and name of the Form tags used in the website, we can find the website category.

\section{Image Tags on the web page}

A phishing website can be created by using images instead of using text. The images can be used by taking the snapshot of the legitimate website. In this case, by using the web image matching algorithms [29] we can find the accessing website is using the same size or different size image of legitimate website. To apply the images in the webpage, <image> tags are used.

\section{Href Tags on the web page}

The <href $>$ tag is used to create a link to another document or webpage. We can count the number of href tags of visiting website with the legitimate website and by using this tag, we can check the reference webpage whether it is legitimate page or not. Some times href tag is used to make the link with legitimate webpage and sometimes it redirect the user to not authorised webpage. If the href reference page matches the link with visiting webpage, the site would be legitimate otherwise phishing

\section{Login/Password evaluation}

The phishing websites uses login and password keywords in its webpage. The previous study of phishing has been done on the basis of these two keywords and found that generally banking and e-commerce websites uses these keywords to collect the username and password of the internet user. The legitimate websites which ask the login and password information of the user takes the permission from the security authorities as a Security Certificate to protect the webpage. The HTTPs protocol is assigned for such websites. The phishing websites do not take the permission of security authorities for securing the webpage, so we can check the login and password tags with HTTPs protocol in the website. On the basis of these tags, we can find the accessing website is phishing or legitimate.

\section{Script Tags on the web page}

The phishing site uses the <script> tag to redirect the web user to client-side system. The <script $>$ tag is used to define a client-side script, such as a JavaScript. The <script> element either contains scripting statements, or it points to an external script file through the src attribute. Common uses for JavaScript are image manipulation, form validation and dynamic changes of content. We can find the number of script tags in the accessing websites and can be cross-checked these tags with the legitimate website scripts tags. If the numbers of script tag of accessing and legitimate website are same, we can keep the accessing website record for the observation of phishing.

\section{Link Tags on the web page}

While accessing the link tags of phishing website, it doesn't work or redirect the user to legitimate site which are not directly concerned with the visiting website. A number of link tags are possible like image tag, href tag, form tag, title tag etc. we have examined that while checking all the tags of the webpage, some links does not match the domain name or send the user to not concerning webpage.

Apart from these finding criteria, we can also find the domain age from www.domaintools.com website. By the use of this website, we can find the information about the website, like when it is created and how long it will be exist. Some of the governmental authorities are also working to countermeasure the phishing attack and finding the better solution to protect the user from internet fraud. These authorities have already declared many websites as phishing, so we have taken the help from these authorised sites to increase our database source. Masoumeh Zareapoor et.al. [30] found that feature extraction techniques offer better performance for 
the classification, give stable classification results.

Gaurav et.al. [31] have described, how to identify the phishing websites. In his study, he has suggested following techniques with its advantages and disadvantages:

i. Attribute based anti-phishing techniques, in this technique Attribute-based anti-phishing strategy implements both reactive and proactive antiphishing defenses. The advantage of this technique is that as attribute based anti-phishing considers a lot of checks so it is able to detect more phished sites than other approaches. It can detect known as well as unknown attacks. The disadvantage of this technique is that as multiple checks perform to authenticate site this could result in slow response time.

ii. Genetic Algorithm Based Anti Phishing Techniques, in this technique, genetic algorithms can be used to evolve simple rules for preventing phishing attacks. These rules are used to differentiate normal website from anomalous website. These anomalous websites refer to events with probability of phishing attacks. The advantage of this technique is that it provides the feature of malicious status notification before the user reads the mail. It also provides malicious web link detection in addition of phishing detection. The disadvantage of this technique is that Single rule for phishing detection like in case of URL is far from enough, so we need multiple rule set for only one type of URL based phishing detection.

iii. An Identity Based Anti Phishing Techniques, This technique follows mutual authentication methodology where both user and online entity validates each other's identity during handshake. It is an anti-phishing technique that integrates partial credentials sharing and client filtering technique to prevent phishing attack from easily masquerading as legitimate online entities. The advantage of this technique is that it provide mutual authentication for server as well as client side. Using this technique, user does not reveal his credential password in whole session except first time when the session is initialized. The disadvantage of this technique is that in identity based anti-phishing, if a hacker gain access to the client computer and disable the browser plug-in then method will be compromise against phishing detection.

iv. Character Based Anti Phishing Approach, in this technique character based anti-phishing technique uses characteristics of hyperlink in order to detect phishing links. The advantage of this concept is that it not only detect known attacks, but also is effective to the unknown ones but the disadvantage of this concept is that it may result false positives, since using dotted decimal IP addresses instead of domain names may be desirable in some special circumstances.

v. Content Based Anti-Phishing Approach, According to this concept, the phishing web pages are active only for short period of time and therefore will acquire low rank during internet search and this becomes basis for content based anti-phishing approach.

\section{PRoposed ARCHITECTURE AND Working ENVIRONMENT}

To test the proposed anti-phishing system, the add-on tool should be applied at the educational institution because of at an educational institution, there are different subjective departments and educated persons who can produce accurate result and can help in the research study and analysis of the anti-phishing tool's performance. We have applied an anti-phishing test bed at the autonomous college. For checking the tool's performance, a test bed setup is applied at the college in which computers were configured using Intel Core I3 CPU $4300 @ 1.80 \mathrm{GHz}$ processor. Each PC was configured with 2 GB RAM and 80 GB hard disk. We have taken same configuration computers to avoid network latency. In the Figure 1, an diagram of the network structure at the college is demonstrated. In this network, different department computers are attached with the dedicated assigned server which is directly connected with a main server. The anti-phishing tool is loaded at both client and server side. When the user at client side computer access the website, add-on start the functioning and gives the messages according to the website type. As per the user's answer given to the addon tool, the results get stored at the dedicated assigned server. The functioning of the assigned server is to collect the information received from client side computer and send it to main server for the analysis of the tool's performance. This result comes from all the departments to the dedicated assigned server. The result then analysed at the main server. At the main server, WEKA (Waikato Environment for Knowledge Analysis), a data mining analysis software is loaded. The WEKA is designed to solve the data mining algorithm issue, which is an open Java source code that includes implementations of different methods for several different data mining tasks such as clustering, classification, association rules and regression analysis.

At the college campus, all the department computers were connected with the wireless LAN. The set-up of the LAN system was as under mentioned:

1. Due to a large number of computers connected with the LAN, the client/server model is applied at the college.

2. The Bus and Tree topology is configured on the LAN.

3. The college was connected with the leased line of Internet with the additional hardware support of Router, Switches and Access Points.

4. Dedicated servers comprise a File, Print Server, Administration Database server and Applications servers are assigned on the network. 
5. Switches are used to connect different department computers, administrative section, library, account section and general sections of the college.

For installing and applying the add-on tool, we have taken the help from a task manager and set of computer workers, each of which is responsible for checking and evaluating the tool. During the test at the site, the task manager has installed the anti-phishing add-on on web browser of all the computers. The task manager informs all the person of different departments to use different websites at their computer and send the feedback information which the web browser asks to the user.

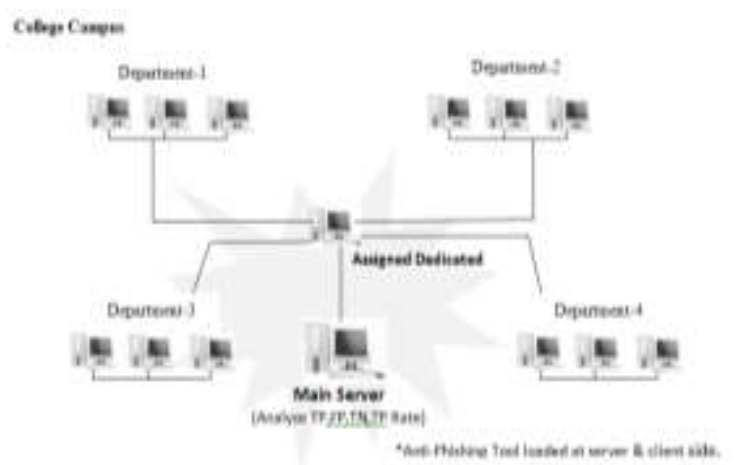

Fig.1. Anti-Phishing System Model at Educational Institute

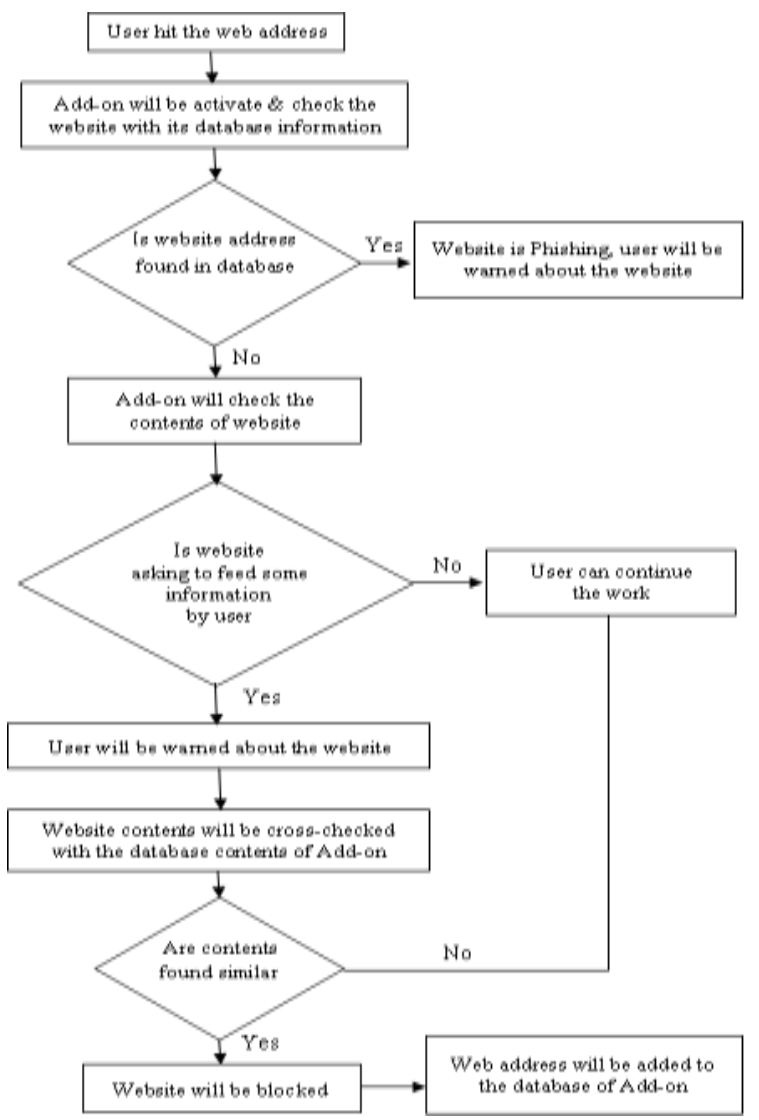

Fig.2. The System Model for Anti-Phishing Approach

We have taken around three hours for 10 different days to collect the result data from the add-on tool. The system tool is tested on January, 2, 7, 12, 17, 22 and 27, 2014 and February 1, 6, 11 and 16, 2014 at the college. During this time, the task manager has collected and sent a batch of new phishing and legitimate websites to the test bed after every 15 minutes. The test bed began the testing of web sites. Each user had opened up the web browser with the installed add-on and given the feedback to the add-on about the web site. As per the accessing website by the user, the add-on tool has collected and tested around 2145 websites in the month of January and February. After receiving the information sent by different users, a database is prepared to analyse the result of the proposed anti-phishing tool. Figure 2 shows the system model of implemented anti-phishing tool at educational institute in which systems are installed at different departments.

The flow of the system model on the basis of system tool functioning is as given below:

\section{EXPERIMENTAL RESULT}

The performance analysis of the anti-phishing tool is tested by getting the response received from antiphishing tools and the user's feedback. During the testing period, around 2145 websites have been tested and 271 websites found suspicious. While checking these suspicious websites with the database information of the anti-phishing tool and Anti-Phishing Working Groups, 249 websites found phishing. The result of per day record is shown in the Table 1. On the basis of this result, the tool's effectiveness can be calculated by

$$
\text { Effectiveness }=\frac{\text { Number of supicious websites }}{\text { Total number of websites }} \times 100
$$

'Number of Phishing websites / Total number of Suspicious Websites' and the accuracy can be calculated by following formula:

$$
\text { Accuracy }=\frac{\text { Number of correct phishing websites }}{\text { Total number of suspicious website }} \times 100
$$

The anti-phishing tool's performance in terms of its effectiveness and accuracy is shown in the following Figure 3.

Table 1. The Results Received from Proposed Anti-Phishing System in Different Days

\begin{tabular}{|l|c|c|c|}
\hline \multicolumn{1}{|c|}{ Days } & $\begin{array}{c}\text { Total } \\
\text { websites }\end{array}$ & $\begin{array}{c}\text { Suspicious } \\
\text { Websites }\end{array}$ & $\begin{array}{c}\text { Websites } \\
\text { Phishing }\end{array}$ \\
\hline 2 January & 252 & 214 & 189 \\
\hline 7 January & 240 & 209 & 186 \\
\hline 12 January & 260 & 233 & 218 \\
\hline 17 January & 176 & 149 & 133 \\
\hline 22 January & 188 & 170 & 151 \\
\hline 27 January & 289 & 264 & 243 \\
\hline 1 February & 205 & 186 & 170 \\
\hline 6 February & 178 & 162 & 151 \\
\hline 11 February & 146 & 131 & 123 \\
\hline 16 February & 211 & 191 & 178 \\
\hline
\end{tabular}




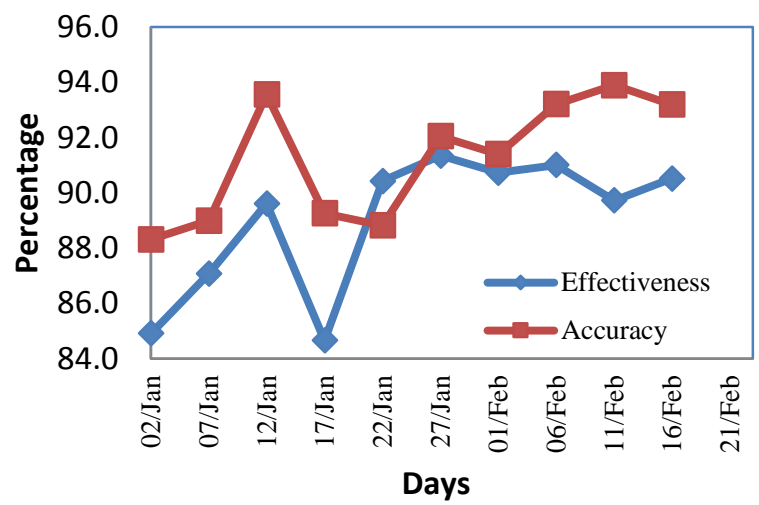

Fig.3. Performance of Anti-Phishing Tool (ePhish) Tested at Educational Institute

The effectiveness of the anti-phishing tool shows around $91.88 \%$ successful result. The tool has detected $2.22 \%$ legitimate websites as phishing. This suspected result is again tested by the system tool by further analysis method and after rectifying the problem; the corrected record is added in the data base of the Add-on. The remaining $5.9 \%$ websites didn't found as legitimate or phishing by the anti-phishing tool, because of $2.6 \%$ websites are designed in other language and $3.3 \%$ websites do not properly formatted. Since the proposed anti-phishing tool is designed for the English language based websites which do not support any other language so the performance of the tool gets down. If we leave the result of any other language websites, the tool's performance reached to around $97.6 \%$.

\section{CONCLUSION}

The Anti-phishing tool with the novel concept is designed and applied at one of the educational institutions to prevent the user from phishing attack. While testing the anti-phishing tool, it is found that the user awareness about the phishing is very essential. If user is not aware of phishing, the spoofing websites can easily steal the personal and confidential information of the user. The anti-phishing tool is showing around 92 percentage successful result for finding the phishing websites. The anti-phishing tool didn't find remaining 8 percentage phishing websites during the test hit because of these websites are designed in other languages or not properly designed and formatted. The division of task in different groups is showing the better result. The phishing problem is growing almost all the areas of information technology sectors. But the problem is severe at the financial and money transactional websites. It is recommended that the anti-phishing tool and its awareness system should be implemented separately for these sectors.

\section{ACKNOWLEDGEMENT}

I thank Dr. Piyush Shukla, Assistant Professor for giving me valuable support and guidance to prepare the manuscript and also to the Principal, BSSS Autonomous College to provide me the working environment for the research work. My college colleagues helped me to find the target websites for the analysis of data and interpretation of the result.

\section{REFERENCE}

[1] Market Share Statistics for Internet Technologies, http://marketshare.hitslink.com/search-engine-marketshare.aspx? qprid=4\&qpcustomd=0, April 2014 .

[2] Egelman S., Cranor L.F. and Hong J. "You've been warned: an empirical study of the effectiveness of web browser phishing warnings. In $\mathrm{CHI}$ '08: Proceeding of the twenty sixth annual SIGCHI conference on Human factors in computing systems New York, NY, USA, ACM 2008, pp. 1065-1074.

[3] Jiang Hansi, Zhang Dongsong, Yan Zhijun, "A Classification Model for Detection of Chinese Phishing eBusiness Websites", PACIS Proceedings. Paper 152, 2013.

[4] Zhuang Weiwei, Jiang Qingshan, Xiong Tengke, "An Intelligent Anti-phishing Strategy Model for Phishing Website Detection", IEEE Computer Society, 32nd International Conference on Distributed Computing Systems Workshops, 2012.

[5] Balamuralikrishna T., Raghavendrasai N., Satya Sukumar M., "Mitigating Online Fraud by Ant phishing Model with URL \& Image based Webpage Matching", International Journal of Scientific \& Engineering Research Volume 3, Issue 3, March-2012, pp.1-6.

[6] Madhuri S. Arade, Bhaskar P.C., Kamat R.K., "Antiphishing Model with URL \& Image based Webpage Matching”, International Conference \& Workshop on Recent Trends in Technology, (TCET), Proceedings published in International Journal of Computer Applications ${ }^{\circledR}$ (IJCA), 2012, pp 18-23.

[7] Aburrous Maher, Hossain M.A., Dahal Keshav, Thabatah Fadi, "Modelling Intelligent Phishing Detection System for e-Banking using Fuzzy Data Mining", IEEE Computer Society, International Conference on CyberWorlds, 2009, pp. 265-272.

[8] Zhuang W., Ye Y., Li T., Jiang Q. "Intelligent phishing website detection using classification ensemble Systems Engineering Theory \& Practice", Volume 31(10), 2011, P2008-2020.

[9] JungMin Kang, Lee DoHoon, "Advanced White List Approach for Preventing Access to Phishing Sites" International Conference on Convergence Information Technology (ICCIT 2007), 2007, pp.491-496.

[10] Abbasi Ahmed, Fatemeh "Mariam" Zahedi and Yan Chen, "Impact of Anti-Phishing Tool Performance on Attack Success Rates", $10^{\text {th }}$ IEEE International Conference on Intelligence and Security Informatics (ISI) Washington, D.C., USA, June 11-14, 2012.

[11] Abbasi A. and Chen H. "A Comparison of Fraud Cues and Classification Methods for Fake Escrow Website Detection," Information Technology and Management, Vol. 10(2), 2009, pp. 83-101.

[12] Bansal G., Zahedi F.M., and Gefen D., "The Impact of Personal Dispositions on Information Sensitivity, Privacy Concern and Trust in Disclosing Health Information Online," Decision Support Systems, Vol. 49(2), 2010, pp. 138-150.

[13] Chen Y., Zahedi F.M., and Abbasi A., "Interface Design Elements for Anti-phishing Systems," In Proc. Intl. Conf. Design Science Research in Information Systems and Technology, 2011, pp. 253- 265.

[14] Grazioli S. and Jarvenpaa S.L., "Perils of Internet Fraud: 
An Empirical Investigation of Deception and Trust with Experienced Internet Consumers," IEEE Trans. Systems, Man, and Cybernetics Part A, vol. 20(4), 2000, pp. 395410.

[15] Martin A., Anutthamaa Na.Ba., Sathyavathy M., Marie Manjari Saint Francois, Dr. Venkatesan Prasanna, "A Framework for Predicting Phishing Websites Using Neural Networks", IJCSI International Journal of Computer Science Issues, Vol. 8, Issue 2, March 2011, pp. 330-336.

[16] Aburrous Maher, Hossain M.A., Dahal Keshav, Thabtah Fadi, "Intelligent phishing detection system for e-banking using fuzzy data mining", Expert Systems with Applications: An International Journal Vol. 37 Issue 12, 2010.

[17] Zhang H., Liu G., Chow T., Liu W., "Textual and Visual Content-Based Anti-Phishing: A Bayesian Approach", IEEE Transactions on Neural Networks, 22(10), 2011, pp. 1532-1546.

[18] Herzberg A. and Jbara A. "Security and identification indicators for browsers against spoofing and phishing attacks", ACM Transactions on Internet Technology, 8(4) 2008, pp. 1-36.

[19] Prakash P., Kumar M., Kompella R.R., and Gupta M., "Phish-Net: predictive blacklisting to detect phishing attacks," in IEEE INFOCOM Proceedings. San Diego, California, USA: IEEE, March, 2010, pp. 1-5.

[20] Garera S., Provos N., Chew M., and Rubin A. D., "A framework for detection and measurement of phishing attacks." Alexandria, Viriginia, USA: ACM, 2007, pp. 18.

[21] Dunlop Matthew, Groat Stephen, and Shelly David, "GoldPhish: Using Images for Content-Based Phishing Analysis", The Fifth International Conference on Internet Monitoring and Protection, IEEE Computer Society, 2010, pg. 123-128.

[22] Chou N., Ledesma R., Teraguchi Y., Boneh D., and Mitchell J. "Client-side defense against web-based identity theft". In 11th Network and Distributed System Security Symposium (NDSS), 2004

[23] Ross B., Jackson C., Miyake N., Boneh D., and Mitchell J. "Stronger Password Authentication Using Browser Extensions", in 14th Usenix Security Symposium, 2005

[24] Microsoft. Sender ID Framework Overview (2005). http://www.microsoft.com

[25] Yahoo. Yahoo! Anti-Spam Resource Center (2006). http://antispam.yahoo.com

[26] Hara M., Yamada A., and Miyake Y., "Visual similaritybased phishing detection without victim site information." Nashville, Tennessee, USA: IEEE, April 2009, pp. 30-36.

[27] Zhang Y., Egelman S., Cranor L., and Hong J., "Phinding phish: Evaluating Anti-Phishing tools," in Proceedings of the $14^{\text {th }}$ Annual Network \& Distributed System Security Symposium, San Diego, California, USA, 2007.

[28] Zhang Y., Hong J., and Cranor L., "CANTINA: A Content-Based approach to detecting phishing web sites," in Proceedings of the 16th international conference on Worldwide Web. Banff, Alberta, Canada: ACM, 2007, pp. 639-648.

[29] Dunlop Matthew, Groat Stephen, and Shelly David, "GoldPhish: Using Images for Content-Based Phishing Analysis", The Fifth International Conference on Internet Monitoring and Protection, IEEE Computer Society, 2010.

[30] Masoumeh Zareapoor, Seeja K. R, "Feature Extraction or Feature Selection for Text Classification: A Case Study on Phishing Email Detection", International Journal of Information Engineering and Electronic Business
(IJIEEB), Vol. 7, No. 2, PP.60-65, March, 2015

[31] Gaurav, Mishra Madhuresh, Jain Anurag, "Anti-Phishing Techniques: A Review, International Journal of Engineering Research and Applications (IJERA), Vol. 2, Issue 2, Mar-Apr 2012, pp.350-355.

[32] Minal Chawla, Siddarth Singh Chouhan, "A Survey of Phishing Attack Techniques, International Journal of Computer Applications, Vo.1 93, No. 3, 2014 pp. 32-35.

\section{Authors' Profiles}

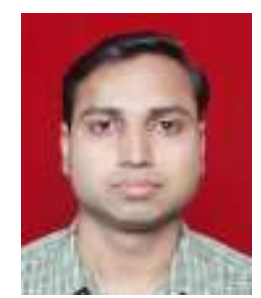

Mr. Rajendra Gupta has completed Master degree in Information Technology, M.Phil and pursing Ph.D. (Computer Science). He has published 6 research papers in International Journals, 3 research papers in National Conferences and completed one research project. At present he is working as an Assistant Professor in Department of Computer Applications, BSSS Autonomous College, Bhopal for last ten years and Member of the various Academic Bodies.

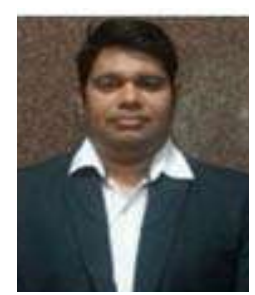

Dr. Piyush K. Shukla received his Bachelor's degree in Electronics \& Communication Engineering, LNCT in 2001, Bhopal, M. Tech (Computer Science \& Engineering) in 2005 from SATI, Vidisha, Ph.D. (Computer Science \& Engineering) in 2013 from RGPV, Bhopal. M.P. India. He is a member of IACSIT. He has published more than 15 papers in reputed International Journals and 10 papers in International Conferences. At present, he is working as an Assistant Prof. in Department of Computer Science \& Engineering, UIT-RGPV, Bhopal Since July 2007.

How to cite this paper: Rajendra Gupta, Piyush Kumar Shukla,"Experimental Analysis of Browser based Novel AntiPhishing System Tool at Educational Level", International Journal of Information Technology and Computer Science(IJITCS), Vol.8, No.2, pp.78-84, 2016. DOI: 10.5815/ijitcs.2016.02.10 